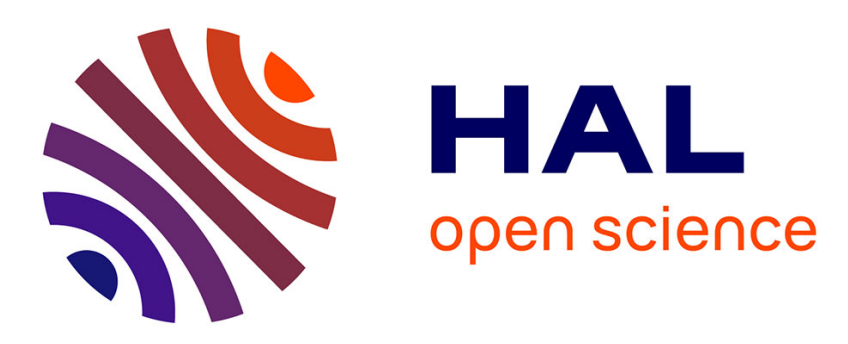

\title{
Proteome oxidative modifications and impairment of specific metabolic pathways during cellular senescence and aging
}

Marie-Paule Hamon, Emad K Ahmed, Martín Baraibar, Bertrand Friguet

\section{- To cite this version:}

Marie-Paule Hamon, Emad K Ahmed, Martín Baraibar, Bertrand Friguet. Proteome oxidative modifications and impairment of specific metabolic pathways during cellular senescence and aging. Proteomics, 2019, pp.1800421. 10.1002/pmic.201800421 . hal-02361135

\section{HAL Id: hal-02361135 \\ https://hal.sorbonne-universite.fr/hal-02361135}

Submitted on 13 Nov 2019

HAL is a multi-disciplinary open access archive for the deposit and dissemination of scientific research documents, whether they are published or not. The documents may come from teaching and research institutions in France or abroad, or from public or private research centers.
L'archive ouverte pluridisciplinaire HAL, est destinée au dépôt et à la diffusion de documents scientifiques de niveau recherche, publiés ou non, émanant des établissements d'enseignement et de recherche français ou étrangers, des laboratoires publics ou privés. 


\section{Proteome oxidative modifications and impairment of specific metabolic pathways during cellular senescence and aging}

Marie-Paule Hamon a , Emad K. Ahmed ${ }^{\text {b }}$, Martín A. Baraibar ${ }^{\mathrm{c}}$ and Bertrand Friguet ${ }^{\text {a1 }}$

a Sorbonne Université, CNRS, INSERM, Institut de Biologie Paris-Seine, Biological Adaptation and Ageing, B2A-IBPS, F-75005 Paris, France ${ }^{\mathrm{b}}$ Biochemistry Department, Faculty of Science, Ain Shams University, Cairo, Egypt; ${ }^{\mathrm{C}}$ OxiProteomics SAS, F-75005 Paris, France.

${ }^{1}$ Corresponding author

E-mail: bertrand.friguet@sorbonne-universite.fr

Keywords: cellular aging, energy metabolism, myoblasts, protein oxidation, WI-38 fibroblasts

Abbreviations: Acetyl-CoA: acetyl coenzyme A; AGE: advanced glycation end-product; HNE: 4hydroxyl-2-nonenal; MDA: malondialdehyde; MS: mass spectrometry; NAD: nicotinamide adenine dinucleotide; NADPH: nicotinamide adenine dinucleotide phosphate; NRF-2: Nuclear factor (erythroid-derived 2)-like 2; RMI: Relative Modification Index; ROS: reactive oxygen species; TCA: tricarboxylic acid; Vol: volume; 2DPAGE: two dimensional polyacrylamide gel electrophoresis 


\begin{abstract}
Accumulation of oxidatively modified proteins is a hallmark of organismal aging in vivo and of cellular replicative senescence in vitro. Failure of protein maintenance is a major contributor to the age-associated accumulation of damaged proteins that is believed to participate to the agerelated decline in cellular function. In this context, quantitative proteomics approaches, including 2D-gel electrophoresis based methods, represent powerful tools for monitoring the extent of protein oxidative modifications at the proteome level and for identifying the targeted proteins, also referred as to the “oxi-proteome”. Our previous studies have identified proteins targeted by oxidative modifications during replicative senescence of human WI-38 fibroblasts and myoblasts and have been shown to represent a restricted set within the total cellular proteome that fall in key functional categories, such as energy metabolism, protein quality control and cellular morphology. To provide mechanistic support into the role of oxidized proteins in the development of the senescent phenotype, untargeted metabolomic profiling was also performed for young and senescent myoblasts and fibroblasts. Metabolomic profiling was indicative of energy metabolism impairment in both senescent myoblasts and fibroblasts, suggesting a link between oxidative protein modifications and the altered cellular metabolism associated with the senescent phenotype of human myoblasts and fibroblasts.
\end{abstract}




\section{Introduction}

Among the main features of aging are failures in proteome homeostasis resulting in the accumulation of abnormal proteins such as oxidatively modified proteins. These proteostasis impairments are due to a decreased efficiency of systems involved in protein quality control and stress response together with an increased occurrence of damaging post-translational protein modifications ${ }^{[1]},{ }^{[2]},{ }^{[3]}$.

Protein post-translational modifications are numerous and various ${ }^{[4]}$. Some are helpful and others are deleterious for the cell, particularly the irreversible ones that are often associated with diseases and aging. By altering protein structure and function, and consequently cell homeostasis, these modifications are even more harmful in the aging process as they occur in a context of maintenance systems displaying declining abilities ${ }^{[5]},{ }^{[6]}$.

Oxidation is one of the most common post-translational modifications of proteins. This phenomenon is due to reaction of protein with reactive oxygen species (ROS). Unless repaired or removed from cells, oxidized proteins accumulate and threaten cell viability. The important role of protein oxidation in aging relies on the fact that increase in oxidized proteins levels is observed during the aging process ${ }^{[7][8]}$. In this case, as in a number of age related diseases, ROS reach levels overwhelming cellular defenses capacities creating an oxidative stress and triggering proteome damage. Enabling protein homeostasis to cope with oxidative stress, particularly at the level of protein quality control, could be one of the major ways to fight against the senescence associated oxidative damages ${ }^{[9]}$.

The irreversible oxidative damages to proteins is manifested mainly by the addition of carbonyl groups, either by direct oxidation of amino acids, by conjugation with lipid peroxidation products such as 4-hydroxyl-2-nonenal (HNE), or by glycoxidation generating AGE (advanced glycation end-product) ${ }^{[10]}$, ${ }^{[11]}$. The carbonylation of proteins is the result of a process by which aldehydes or ketones are introduced by oxidation. It occurs on the polypeptide chain and on the nucleophilic or redox sensitive side chains of amino acids. Because of their constitution some 
amino acids are more prone to oxidative carbonylation than others. This is the case of proline, arginine, lysine, threonine and histidine ${ }^{[12]}{ }^{[13]}$.

Lipid peroxidation is another protein damaging modification inducer that refers to the oxidation of polyunsaturated lipids following a radical chain reaction process ${ }^{[14]}$. This results initially in a wide range of oxidized products, including lipid hydroperoxides which will then give rise to various aldehydes such as HNE and MDA (malondialdehyde). The aldehydes resulting from this oxidative mechanism are stable products capable of attacking protein targets relatively far from their place of formation, on which they form Michael adducts. Particularly deleterious, these carbonyl adducts are formed on histidine, cysteine and lysine residues.

The modification of the proteins can also result from protein glycoxidation occurring on a previously glycated protein ${ }^{[15]}$. This cascade of events begins with a process of glycation, namely the formation of a Schiff base by interaction between the aldehyde of a glucose and the free amino function of an amino acid, usually a lysine or arginine. Until then the process is reversible. However, if it continues, the Schiff base undergoes an Amadori rearrangement which stabilizes the condensation of the saccharide residue on the protein chain. These Amadori products can then be the target of glycoxidation reactions responsible for the formation of protein carbonyls and/or for the constitution of advanced glycation end products, i.e. the deleterious AGEs ${ }^{[16]}$. In addition, protein can also be modified by reaction of arginine or lysine amino groups with reactive dicarbonyl compounds as glyoxal and methylglyoxal that gives rise to the formation of such end products as carboxymethylysine, carboxyethyllsine or imidazolone derivatives.

Accumulation of carbonylated proteins is observed during various oxidative stresses, during aging and in many pathologies. So much so that carbonylation can be considered as a hallmark of oxidative stress but the proteins that are targeted by this or other damaging modifications have not been identified in most cases. Indeed, the identification of damaged protein targets is expected not only to define potential biomarkers but also to give insight into the mechanisms by which these damaged proteins accumulate and may contribute to cellular dysfunction. Interestingly, the identification of proteins increasingly carbonylated or modified by AGE or HNE during aging, age- 
related diseases and cellular senescence has shown that they represent a restricted set of the cellular proteome that fall in key functional categories, such as protein quality control, cellular morphology and energy metabolism ${ }^{[17]}$. Several proteins were recently identified in a proteomic study demonstrating in WI-38 human fibroblasts a strong interplay between replicative senescence, selenium and selenoproteins which are known to play a role in antioxidant defense and redox homeostasis ${ }^{[18]}$. Of note, the restricted sub-sets of modified proteins in senescent fibroblasts and myoblasts are different although an overlap of four proteins, involved in protein quality control, cellular morphology and energy metabolism, was found for both cell types ${ }^{[19]}{ }^{[20]}$. In this paper, these findings are described and further discussed in view of the potential impact of oxidatively modified proteins on the altered metabolism of both senescent myoblasts and fibroblasts, with a specific focus on energy metabolism. Since senescence, as well as ageing have to be approached with a multimarker process ${ }^{[21]}$, this issue was addressed at both proteomic and metabolomic levels in these two cell types. Indeed, while informative publications studied replicative senescence in fibroblasts with either a proteomic ${ }^{[22]}$ or a metabolomic ${ }^{[23]}{ }^{[24]}$ points of view, here we used both approaches to analyze the effects of replicative senescence in human fibroblasts and myoblasts.

\section{Protein oxidative modifications in senescent fibroblasts and myoblasts are mainly observed for protein involved in energy metabolism, quality control, stress response and cytoskeleton organization}

Since replicative senescence has proven to be a model of cellular aging in vitro ${ }^{[17]}$, we performed studies using young and senescent fibroblasts and myoblasts in order to address the role of protein oxidative modifications during cellular aging ${ }^{[25]}{ }^{[19]},{ }^{[20]},{ }^{[21]}$. These studies were particularly focused on highlighting the affected cellular pathways through the identification of proteins presenting increased oxidative modification levels during replicative senescence.

Immunodetection of carbonylated, HNE- and AGE- modified proteins was performed after 1D and 2D gel electrophoresis on young and senescent human WI-38 fibroblasts and skeletal muscle satellite cells ${ }^{[19]}{ }^{[20]}$. Increased levels of these oxidative modifications were observed 
during replicative senescence in both cell types, encouraging the identification of the proteins targeted by these modifications and, hence, the potential mechanisms by which this accumulation of modified proteins could affect cellular functions.

For this purpose, total protein extracts from young and senescent cells were resolved by twodimensional electrophoresis (2DPAGE). For each sample, two gel series were prepared in parallel, one for immunodetection of carbonylated, AGE- or HNE-modified proteins and the second for silver nitrate or Coomassie colloidal blue staining to visualize the total protein profile, equal loading control, and subsequent identification by MS (mass spectrometry) analysis. Interestingly, the pattern of the modified proteins was not superimposable with the pattern obtained for the total protein staining, indicating that certain proteins represent preferential targets for these detrimental modifications.

According to these analyses, 30 and 28 protein spots were respectively found increasingly modified during replicative senescence in fibroblast and myoblasts (Tables 1 and 2). All the proteins identified and listed in the tables 1 and 2 have been assigned a Mascot score greater than 56, i.e. significant at $\mathrm{p}<0.05$. In senescent fibroblasts, about half of the modified proteins are localized in mitochondria while, in senescent myoblasts, only one modified protein belongs to this organelle. Hence, protein oxidation accompanying replicative senescence is impacting differently the various cellular compartments depending on the cell type. In senescent fibroblasts, although the most important modification process is lipid peroxidation through the formation of HNE adducts, the number of protein targets for one or more of the three ways of oxidative modification is quite the same. In contrast, it is the lipid peroxidation that has the lowest impact in myoblasts.

In both cell types, most of the oxidatively modified proteins identified are found to fall into three main categories: energy metabolism, quality control-stress response and cytoskeleton organization (Figure $1 \mathrm{~A}$ and B). Regarding quality control and stress response, about $40 \%$ of the spotted proteins act interestingly on oxidative stress. However, the similarities stop here. Only four modified proteins are common to senescent fibroblasts and myoblasts, i.e. G6PD, HSPA8, CCT6A and vimentin (Fig 2 A and B) and, except for CCT6A, they are not even affected in the same way. 
For instance, in fibroblasts, vimentin is particularly affected during senescence as it simultaneously suffers from direct carbonylation, as well as HNE and AGE adductions. Vimentin has been shown to be prone to binding of $\mathrm{HNE}^{[26]}$ and it has been observed that senescent primary fibroblasts express on their cell surface vimentin presenting lipid peroxidation adducts ${ }^{[27]}$. Indeed vimentin is subject to various non-enzymatic modifications, mostly oxidative in nature, which may be involved in the regulation of the vimentin network under stress conditions. These modifications may become particularly influential in senescent fibroblasts ${ }^{[28]}$. Interestingly, it has been shown that vimentin delays senescence and promote immortalization in primary mouse embryo fibroblasts [29].

Upon examination of the oxidatively modified proteins detected in the energy metabolism pathways, quality control-stress response and cytoskeleton of senescent fibroblasts and myoblasts, we noted that they are not involved in the same mechanisms of these three cellular functions (Figure 3 A and B). Regarding energy metabolism of senescent fibroblasts, oxidatively modified proteins are essentially associated with the TCA (tricarboxylic acid) cycle (SDHA, DLD, OGDH, MDH2 proteins), the mitochondrial function (HSD17B10, SDHA, NDUFS2, UQCRC1, OGDH proteins), the oxidative phosphorylation (SDHA, NDUFS2, UQCRC1 proteins) and the fatty acid $\beta$ oxidation (ALDH2, ALDH1A1 proteins). This result is consistent with the important proportion of oxidized proteins found in mitochondria of senescent fibroblasts. In senescent myoblast, energy metabolism is mainly affected by oxidative modifications when it comes to glycolysis (ENO1, TPI1, PGAM1, ALDOA, GAPDH proteins).

Concerning the quality control and stress response, although the modified proteins are not the same in senescent fibroblast compared to senescent myoblasts, the impacted pathways are more similar, that is the unfolded protein response (HSPA8, CALR in fibroblasts; HSPA8, HSP90B1 in myoblasts), the sirtuin signaling pathway (SDHA, G6PD, NDUFS2 in fibroblasts; PGAM1, G6PD, PDK1 in myoblasts), the endoplasmic reticulum stress pathway (CALR in fibroblasts; HSP90B1 in myoblast). However, senescent fibroblasts present a greatest effect on proteins associated with the NRF-2 (Nuclear factor (erythroid-derived 2)-like 2)-mediated oxidative stress response (ERP29, ACTB in fibroblasts; STIP1 in myoblasts) while in senescent myoblasts oxidized proteins are more 
highlighting the protein ubiquitination pathway (HSPA8, HSP90B1, PSMC6 in myoblasts; HSPA8, PSMA2 in fibroblast) and also the glutathione redox reactions I (PRDX6) that is only emerging in this cell type.

In fibroblasts like in myoblasts, replicative senescence is associated with the oxidative modification of a number of proteins mostly belonging to the same main cellular functions. However, within these three functions (cytoskeleton organization, quality control-stress response and energy metabolism), it is not the same pathways that are targeted by the oxidative protein modifications observed in fibroblasts and myoblasts. Therefore it can be expected that the oxidatively modified protein accumulation accompanying replicative senescence may differently affect fibroblasts and myoblasts.

Regarding cytoskeleton organization, senescent fibroblasts notably exhibit HNE adducts on actin and tubulin (Table 1). Oxidation of both proteins have yet been highlighted with an ensuing cytoskeletal disruption ${ }^{[30]}$ and inducing damages in the nervous system cytoskeleton ${ }^{[31]}$. In contrast, protein oxidative modifications observed in senescent myoblasts are not directly acting on actin and tubulin but on proteins such as desmin, caldesmon, gelsolin, lamin A, and fascin (Figure 2B) known to regulate three major components of the cytoskeleton architecture, i.e. actin, tubulin and intermediate vimentin filaments ${ }^{[32]}$.

Concerning the quality control and stress response, the pathways using proteins affected by oxidative modifications during senescence of fibroblasts and myoblasts are quite similar (Figure 3). However we noted that the protein oxidation observed in the protein ubiquitination pathway seems more pronounced in myoblasts. Ubiquitinated proteins are intended for degradation by the proteasome and several studies suggest that oxidation products could inhibit the proteasome through a blockage of substrates accessibility ${ }^{[33]}{ }^{[34]}$.

Detoxification systems are other defense mechanisms used by cells against oxidative stress. According to Ingenuity Pathway Analyses (Figure 3), the “Glutathione Redox Reactions” canonical pathway seems only affected in senescent myoblasts with PRDX6 being HNE-modified. Conversely, the "NRF2-mediated Oxidative Stress Response" canonical pathway seems more affected in senescent fibroblasts than in senescent myoblasts with ERP29 and ACTB versus STIP1 
being oxidatively modified, respectively. This observation may be linked with the high number of oxidized proteins found in the mitochondria of senescent fibroblasts since NRF-2 is also involved in the regulation of mitochondrial biogenesis ${ }^{[35]}$.

However, it is at the energy metabolism level that the disparities between senescent fibroblasts and myoblasts are the most obvious (Figure 3). Oxidatively modified proteins identified in senescent fibroblasts are principally acting in metabolic processes localized in mitochondria, i.e. TCA cycle, oxidative phosphorylation and fatty acid $\beta$-oxidation. By altering the function of proteins targeted by oxidative modifications, these modifications might have caused a dysfunction of the quality control systems and the mitochondrial energetic metabolism that could result in a stimulation of the ROS production thus being able to induce a vicious circle. Such an effect has already been observed for the complex I of the respiratory chain ${ }^{[36]}$. In contrast, it has been shown that fibroblasts adopt a more glycolytic state when they become senescent ${ }^{[37]}$.

On the contrary, glycolysis is particularly impacted by protein oxidative modifications in senescent myoblasts. Indeed five enzymes belonging to this pathway are modified and, among them, two are notably affected, namely ALDOA and ENO1 that present the three forms of oxidative modifications (Table 2). Thus, in the case of myoblasts, senescence appears to impair glucose oxidation at the level of anaerobic glycolysis.

Interestingly G6PD was found oxidatively modified in both senescent fibroblasts and myoblasts (Figure 2). G6PD is known to supply reducing equivalents by regenerating NADPH (nicotinamide adenine dinucleotide phosphate) levels as well as pentose phosphates for fatty acid and nucleic acid synthesis. Acting in the oxidative branch of the pentose phosphate pathway, G6PD is a major source of NADPH and therefore a key enzyme for maintaining redox homeostasis ${ }^{[38]}$. The protective role of G6PD against the deleterious effects of ROS has been observed on several occasions ${ }^{[39]}{ }^{[38]}$. Thus, with the oxidative modification of this enzyme, senescent fibroblasts like senescent myoblasts could lose here another way to deal with the age-associated increase of oxidative stress.

We have also noted that many mitochondrial dehydrogenases were found oxidized in senescent fibroblasts, namely ALDH4A1, ALDH2, HSD17B10, MDH2, SDHA, DLD, OGDH, 
NDUFS2 and IVD (Table 1). The oxidative modification of these enzymes may also have an impact on their functioning. For example, ALDH4A1 and ALDH2 are respectively involved in acetaldehyde and glutamate semi-aldehyde detoxification and their adduction with lipid peroxidation products could increase the load of reactive aldehyde in senescent fibroblasts. In addition, HSD17B, DLD and NDUFS2, as component of NADH dehydrogenase, complex I of the respiratory chain, can influence the redox homeostasis through the dysregulation of the couple $\mathrm{NADH} / \mathrm{NAD}^{+}$as observed for both myoblasts ${ }^{[20]}$ and fibroblasts (Figure 5) although in opposite directions. Indeed, changes of the $\mathrm{NAD}^{+} / \mathrm{NADH}$ ratio can modulate mitochondrial quantity and quality ${ }^{[40]}$. Morever, $\mathrm{NAD}^{+}$is a co-substrate for sirtuins and a critical molecule in metabolism and redox signalling ${ }^{[41]}$.

\section{Metabolome analyses show differential effects on energy metabolism in senescent myoblasts} and fibroblasts.

Metabolites profiling analysis was performed by Metabolon, Inc. as previously described ${ }^{[42]}$. Cellular pellets $\left(10^{6}\right.$ cells) from six different batches from young and senescent fibroblasts were accessioned into the Metabolon LIMS system. Raw data was extracted, peak-identified and QC processed using Metabolon's hardware and software ${ }^{[43]}$. Welch’s two-sample t-test was used to identify biochemicals that differed significantly between experimental groups.

Differential effects on energy metabolism were observed in myoblasts and fibroblasts undergoing replicative senescence. As previously reported ${ }^{[20]}$, glycolytic intermediates such as glucose-6-phosphate, fructose-6-phosphate, and the isobar containing fructose 1,6-diphosphate, glucose 1,6-diphosphate, and myo-inositol 1,4 or 1,3-diphosphate were decreased in senescent myoblasts, which is indicative of a decreased metabolism of glucose via glycolysis. Interestingly, 6 enzymes involved in glycolysis were found to be increasingly modified in senescent myoblasts: aldolase, triosephosphate isomerase, glyceraldehyde 3-phosphate dehydrogenase, phosphoglycerate mutase, enolase and pyruvate kinase ${ }^{[20]}$. The oxidative modification of these enzymes is concomitant with a decreased glucose oxidation and an increased NAD+/NADH ratio, reflecting a 
decreased cellular reducing potential ${ }^{[20]}$. Since the functionality of the mitochondrial respiratory chain was not affected in human myoblasts during replicative senescence, the decreased glucose oxidation we observed is most likely due to an impairment in glycolysis and/or TCA cycle. Oxidative modifications of enzymes involved in glycolysis and the TCA cycle have also been suggested to be an important pathophysiological factor in age-related diseases, such as neurodegenerative diseases ${ }^{[44]}$. Other studies have shown that the inhibition of glycolytic enzymes, such as GAPDH and PGAM by siRNA can induce premature senescence ${ }^{[45]}$.

In contrast to senescent myoblasts, the glycolytic intermediates glucose-6-phosphate (Figure 4), fructose-6-phosphate, and the isobar containing fructose 1,6-diphosphate, glucose 1,6diphosphate, and myo-inositol 1,4 or 1,3-diphosphate (data not shown) were found relatively unchanged in senescent fibroblasts while the downstream intermediates 2-phosphoglycerate, 3phosphoglycerate, and phosphoenolpyruvate are dramatically depleted as compared to young fibroblasts (Figure 4). Together with the observed increase in lactate in senescent fibroblasts, this result suggests an increased glycolytic activity in these cells. Depletion of the pentose phosphate pathway intermediate 6-phosphogluconate also suggests a modified activity of this pathway. Consistent with increased glycolysis, acetyl-CoA (acetyl coenzyme A) levels are elevated in fibroblasts during replicative senescence that likely accounts for the increased TCA intermediates citrate, fumarate, and malate and may indicate a decreased efficiency of the TCA cycle (Figure 5). Furthermore, the increased levels of NADH together with a NAD+ level not significantly modified suggest an impairment of the mitochondrial respiratory chain since NADH produced by the TCA cycle in the mitochondria is utilized by the electron transport chain for ATP generation by oxidative phosphorylation.

\section{Concluding remarks}

Identification of proteins targeted by oxidative modification during replicative senescence of both human fibroblasts and myoblasts showed that they represent a restricted set within the total cellular proteome that fall in key functional categories, such as energy metabolism, quality control- 
stress response and cellular morphology. 30 and 28 protein spots were respectively found increasingly modified during replicative senescence of fibroblasts and myoblasts with about half of the modified proteins localized in mitochondria in senescent fibroblasts while only one modified protein belongs to this organelle in senescent myoblasts. Of note, about $35 \%$ of these proteins that are accumulating as oxidatively modified in senescent fibroblasts and myoblasts have been previously shown to accumulate during aging and age-related diseases ${ }^{[17]}$. During replicative senescence of myoblasts, proteins involved in protein quality control and glycolytic enzymes were the main targets of oxidation and modification with advanced glycation/lipid peroxidation end products. These studies underscore the importance of performing proteomic analyses addressing different aspects, such as expression levels and modifications by carbonylation or glycoxidation, to have a broader view of the age-related changes affecting the cellular proteome. Moreover, for both human fibroblasts and myoblasts, the metabolome analyses are indicative of an energy metabolic switch that is associated with replicative senescence. Interestingly, this energy metabolic switch is different depending on cell type: from glycolysis to fatty acid $\beta$ oxidation in senescent myoblasts and from TCA cycle / oxidative phosphorylation to glycolysis in senescent fibroblasts. Taken together with the proteomic modification analyses showing that oxidatively modified proteins are mainly linked with the TCA cycle and oxidative phosphorylation in senescent fibroblasts while mainly linked with glycolysis in senescent myoblasts, these results suggest that the observed increase of oxidative damage to specific protein targets may have indeed contributed to the senescent associated impairment of energy metabolism specific pathways in both cell types. This was further investigated in senescent myoblasts for which glycolytic flux analyses aimed at monitoring glucose oxidation to $\mathrm{CO}_{2}$ have shown the glycolytic pathway is indeed decreased by about $40 \%$ while the mitochondrial respiration capacity remained the same as compared with young myoblasts ${ }^{[20]}$. Furthermore, the rationale behind the cell type-dependent accumulation of oxidatively modified proteins in specific metabolic pathways during cellular senescence remains to be elucidated. Indeed, beside protein localization and intrinsic susceptibility to oxidation, it may depend on such cell specific features as differences in basal energy metabolism and/or increased susceptibility of catalytically active metabolic enzymes to oxidative modifications. Finally, since 
more than one third of those proteins that are accumulating as oxidatively modified during cellular senescence have also been shown to accumulate during aging and age-related diseases, whether this build-up of specific oxidatively modified proteins is also resulting in the impairment of such metabolic pathways as those evidenced in senescent cells remains to be investigated.

\section{Acknowledgments}

The authors wish to thank Marine Le Boulch for her help in fibroblasts cell culturing. The institutional funding of the laboratory by Sorbonne Université, CNRS and INSERM is acknowledged as well as the past support of EU (FP6-Proteomage and FP7-MyoAge integrated projects) and of the current INSERM cross-cutting program on aging (AGEMED). 


\section{References}

[1] M. A. Baraibar, B. Friguet. Exp Gerontol. 2013,48, 620.

[2] N. Chondrogianni, K. Georgila, N. Kourtis, N. Tavernarakis, E. S. Gonos. Free Radic Biol Med. 2014,75 Suppl 1, S18.

[3] M. P. Hamon, A. L. Bulteau, B. Friguet. Ageing Res Rev. 2015,23, 56.

[4] C. T. Walsh, S. Garneau-Tsodikova, G. J. Gatto, Jr. Angewandte Chemie. 2005,44, 7342.

[5] V. N. S. Vanhooren, A. Navarrete Santos, K. Voutetakis, I. Petropoulos, C. Libert, A. Simm, E. S. Gonos, B. Friguet. Mechanisms of ageing and development. 2015,151, 71.

[6] K. A. Liddy, M. Y. White, S. J. Cordwell. Genome medicine. 2013,5, 20.

[7] E. R. Stadtman. Free Radic Res. 2006,40, 1250.

[8] S. Reeg, T. Grune. Antioxid Redox Signal. 2015,23, 239.

[9] D. Reichmann, W. Voth, U. Jakob. Mol Cell. 2018,69, 203.

[10] M. Le Boulch, E. K. Ahmed, A. Rogowska-Wrzesinska, M. A. Baraibar, B. Friguet. Mech Ageing Dev. 2018,170, 59.

[11] H. Bakala, E. Delaval, M. Hamelin, J. Bismuth, C. Borot-Laloi, B. Corman, B. Friguet. European Journal of Biochemistry. 2003,270, 2295.

[12] C. M. Wong, G. Bansal, L. Marcocci, Y. J. Suzuki. Redox Rep. 2012,17, 90.

[13] M. Cattaruzza, M. Hecker. Circ Res. 2008,102, 273.

[14] A. K. Hauck, D. A. Bernlohr. J Lipid Res. 2016,57, 1976.

[15] G. Vistoli, D. De Maddis, A. Cipak, N. Zarkovic, M. Carini, G. Aldini. Free Radic Res. 2013,47 Suppl 1, 3.

[16] K. Byun, Y. Yoo, M. Son, J. Lee, G. B. Jeong, Y. M. Park, G. H. Salekdeh, B. Lee. Pharmacol Ther. 2017,177, 44.

[17] M. A. Baraibar, L. Liu, E. K. Ahmed, B. Friguet. Oxid Med Cell Longev. 2012,2012, 919832.

[18] G. Hammad, Y. Legrain, Z. Touat-Hamici, S. Duhieu, D. Cornu, A. L. Bulteau, L. Chavatte. Antioxidants (Basel). 2018,7.

[19] E. K. Ahmed, A. Rogowska-Wrzesinska, P. Roepstorff, A. L. Bulteau, B. Friguet. Aging cell. 2010,9, 252. 
[20] M. A. Baraibar, J. Hyzewicz, A. Rogowska-Wrzesinska, A. L. Bulteau, C. Prip-Buus, G. Butler-Browne, B. Friguet. Aging. 2016,8, 3375.

[21] M. Ogrodnik, H. Salmonowicz, V. N. Gladyshev. Aging cell. 2019,18, e12841.

[22] X. Zhang, S. Zhang, X. Liu, Y. Wang, J. Chang, X. Zhang, S. G. Mackintosh, A. J. Tackett, Y. He, D. Lv, R. M. Laberge, J. Campisi, J. Wang, G. Zheng, D. Zhou. Aging cell. 2018,17, e12780.

[23] E. L. James, J. A. Lane, R. D. Michalek, E. D. Karoly, E. K. Parkinson. Sci Rep. 2016,6, 38489.

[24] E. L. James, R. D. Michalek, G. N. Pitiyage, A. M. de Castro, K. S. Vignola, J. Jones, R. P. Mohney, E. D. Karoly, S. S. Prime, E. K. Parkinson. Journal of proteome research. 2015,14, 1854. [25] E. K. Ahmed, C. R. Picot, A. L. Bulteau, B. Friguet. Annals of the New York Academy of Sciences. 2007,1119, 88.

[26] A. Monico, S. Duarte, M. A. Pajares, D. Perez-Sala. Redox Biol. 2019101098.

[27] D. Frescas, C. M. Roux, S. Aygun-Sunar, A. S. Gleiberman, P. Krasnov, O. V. Kurnasov, E. Strom, L. P. Virtuoso, M. Wrobel, A. L. Osterman, M. P. Antoch, V. Mett, O. B. Chernova, A. V. Gudkov. Proc Natl Acad Sci U S A. 2017,114, E1668.

[28] D. Perez-Sala, C. L. Oeste, A. E. Martinez, M. J. Carrasco, B. Garzon, F. J. Canada. Nat Commun. 2015,6, 7287.

[29] G. V. Tolstonog, R. L. Shoeman, U. Traub, P. Traub. DNA and cell biology. 2001,20, 509.

[30] A. Keshavarzian, A. Banan, A. Farhadi, S. Komanduri, E. Mutlu, Y. Zhang, J. Z. Fields. Gut. 2003,52, 720 .

[31] J. Gardiner, R. Overall, J. Marc. Diseases. 2013,1, 36.

[32] D. D. Tang, B. D. Gerlach. Respiratory research. 2017,18, 54.

[33] B. Friguet, L. I. Szweda. FEBS Lett. 1997,405, 21.

[34] A. L. Bulteau, P. Verbeke, I. Petropoulos, A. F. Chaffotte, B. Friguet. J Biol Chem. 2001,276, 45662.

[35] R. A. Gottlieb, D. Bernstein. Cell Calcium. 2016,60, 88.

[36] L. K. Sharma, J. Lu, Y. Bai. Current medicinal chemistry. 2009,16, 1266.

[37] C. D. Wiley, J. Campisi. Cell Metab. 2016,23, 1013. 
[38] A. Stincone, A. Prigione, T. Cramer, M. M. Wamelink, K. Campbell, E. Cheung, V. OlinSandoval, N. M. Gruning, A. Kruger, M. Tauqeer Alam, M. A. Keller, M. Breitenbach, K. M. Brindle, J. D. Rabinowitz, M. Ralser. Biol Rev Camb Philos Soc. 2015,90, 927.

[39] S. Nobrega-Pereira, P. J. Fernandez-Marcos, T. Brioche, M. C. Gomez-Cabrera, A. SalvadorPascual, J. M. Flores, J. Vina, M. Serrano. Nat Commun. 2016,7, 10894.

[40] S. Y. Jang, H. T. Kang, E. S. Hwang. J Biol Chem. 2012,287, 19304.

[41] J. M. Berthiaume, J. G. Kurdys, D. M. Muntean, M. G. Rosca. Antioxid Redox Signal. 2017.

[42] Z. J. Reitman, G. Jin, E. D. Karoly, I. Spasojevic, J. Yang, K. W. Kinzler, Y. He, D. D. Bigner, B. Vogelstein, H. Yan. Proc Natl Acad Sci U S A. 2011,108, 3270.

[43] C. D. Dehaven, A. M. Evans, H. Dai, K. A. Lawton. Journal of cheminformatics. 2010,2, 9.

[44] A. Martinez, M. Portero-Otin, R. Pamplona, I. Ferrer. Brain pathology (Zurich, Switzerland). 2010,20, 281.

[45] H. Kondoh, M. E. Lleonart, J. Gil, J. Wang, P. Degan, G. Peters, D. Martinez, A. Carnero, D. Beach. Cancer research. 2005,65, 177. 
Table 1 -Carbonylated, HNE-modified and AGE-modified proteins in senescent fibroblasts

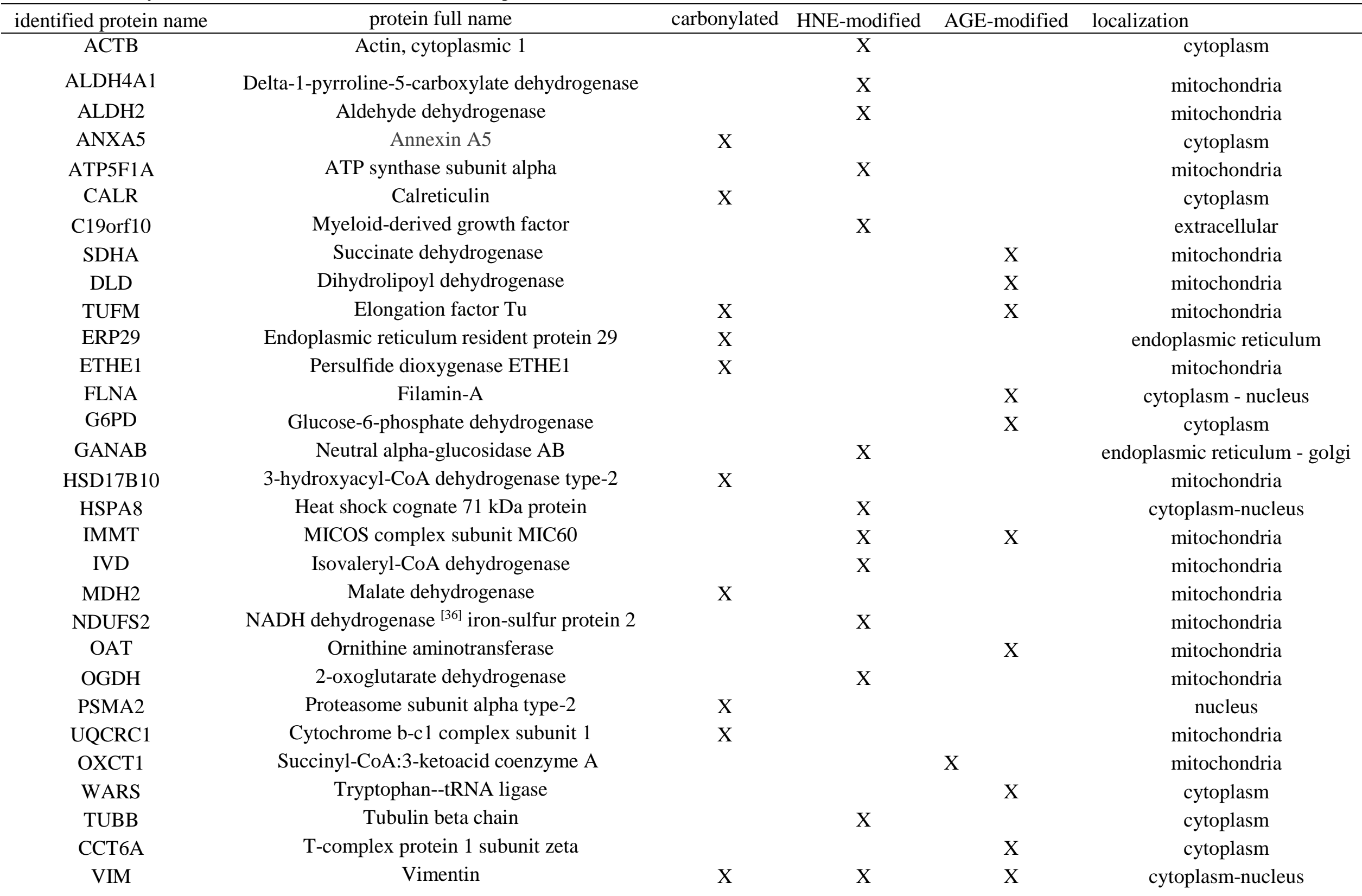

Increasingly modified proteins with RMI (Relative Modification Index) $\geq 1.3$ identified after the experimental procedures described in ${ }^{[19]}$ 
Table 2 - Carbonylated, HNE-modified and AGE-modified proteins in senescent myoblasts

\begin{tabular}{|c|c|c|c|c|c|}
\hline identified protein name & protein full name & carbonylated & HNE-modified & AGE-modified & localization \\
\hline ALDH1A1 & Retinal dehydrogenase 1 & $\mathrm{X}$ & & $\mathrm{X}$ & cytoplasm \\
\hline ALDOA & Fructose-bisphosphate aldolase A & $\mathrm{X}$ & $\mathrm{X}$ & $\mathrm{X}$ & cytoplasm \\
\hline ANXA2 & Annexin A2 & $\mathrm{X}$ & $\mathrm{X}$ & & cytoplasm-extracellular \\
\hline CAP1 & Adenylyl cyclase-associated protein 1 & $\mathrm{X}$ & & & plasma membrane \\
\hline DES & Desmin & & & $\mathrm{X}$ & plasma membrane-nucleus \\
\hline DPYSL2 & Dihydropyrimidinase-related protein 2 & & & $\mathrm{X}$ & cytoplasm \\
\hline HSP90B1 & Heat shock protein $90 \mathrm{kDa}$ beta member 1 & & & $\mathrm{X}$ & endoplasmic reticulum \\
\hline FSCN1 & Fascin & $\mathrm{X}$ & & $\mathrm{X}$ & cytoplasm \\
\hline G6PD & Glucose-6-phosphate dehydrogenase & $\mathrm{X}$ & & & cytoplasm \\
\hline GAPDH & Glyceraldehyde-3-phosphate dehydrogenase & $\mathrm{X}$ & & $\mathrm{X}$ & cytoplasm-nucleus \\
\hline GNB2 & G protein subunit beta-2 & $\mathrm{X}$ & & & cytoplasm-perinuclear \\
\hline GSN & Gelsolin & $\mathrm{X}$ & & $\mathrm{X}$ & cytoplasm-extracellular \\
\hline PLOD2 & Procollagen-lysine,2-oxoglutarate 5-dioxygenase 2 & $\mathrm{X}$ & & & endoplasmic reticulum \\
\hline PLOD3 & $\begin{array}{c}\text { Multifunctional procollagen lysine hydroxylase and } \\
\text { glycosyltransferase LH3 }\end{array}$ & $\mathrm{X}$ & & & $\begin{array}{l}\text { endoplasmic reticulum - } \\
\text { extracellular }\end{array}$ \\
\hline PRDX6 & Peroxiredoxin-6 & & $\mathrm{X}$ & & cytoplasm \\
\hline PSMC6 & $26 \mathrm{~S}$ proteasome regulatory subunit $10 \mathrm{~B}$ & & $\mathrm{X}$ & $\mathrm{X}$ & cytoplasm-nucleus \\
\hline ATIC & Bifunctional purine biosynthesis protein PURH & & & $\mathrm{X}$ & cytoplasm-extracellular \\
\hline STIP1 & Stress-induced-phosphoprotein 1 & & & $\mathrm{X}$ & cytoplasm \\
\hline ССТЗ & T-complex protein 1 subunit gamma & & & $\mathrm{X}$ & cytoplasm \\
\hline ССТ6А & T-complex protein 1 subunit zeta & & & $\mathrm{X}$ & cytoplasm \\
\hline TPI1 & Triosephosphate isomerase & & $\mathrm{X}$ & & cytoplasm \\
\hline VIM & Vimentin & & & $\mathrm{X}$ & cytoplasm-nucleus \\
\hline
\end{tabular}


Figure 1: Network analysis of modified proteins in fibroblasts and myoblasts

Networks obtained using Ingenuity Pathways Analysis (Ingenuity Systems, http://www.ingenuity.com/) for proteins reported to be significantly more oxidized in senescent cells compared to young cells. A) Analysis of modified proteins in fibroblasts. B) Analysis of modified proteins in myoblasts. Proteins in red correspond to proteins that are common to fibroblasts and myoblasts. Information about the analysis of biological functions and pathways as well as network interactions is available at the Ingenuity Pathway Analysis website. A line denotes binding of proteins, whereas a line with an arrow denotes “acts on.” A dotted line denotes an indirect interaction.

Figure 2: Network analysis of modified proteins acting in cytoskeleton organization, quality control-stress response and energy metabolism

Networks obtained using Ingenuity Pathways Analysis (Ingenuity Systems, http://www.ingenuity.com/) for proteins reported to be significantly more oxidized in senescent cells compared to young cells in both fibroblasts and myoblasts. A) Analysis of modified proteins acting in cytoskeleton. B) Analysis of modified proteins acting in quality control and stress response. C) Analysis of modified proteins acting in energy metabolism. Proteins in green correspond to proteins identified in fibroblasts and proteins in orange correspond to proteins identified in myoblasts. Information about the analysis of biological functions and pathways as well as network interactions is available at the Ingenuity Pathway Analysis website. A line denotes binding of proteins, whereas a line with an arrow denotes "acts on.” A dotted line denotes an indirect interaction.

Figure 3: Canonical pathways associated with modified proteins acting in energy metabolism, quality control and stress response 
Modified proteins were grouped by canonical pathways through the use of Ingenuity Pathways Analysis. The bars represent the canonical pathways identified, named in the $x$-axis. The $y$-axis shows the $-\log$ of the $P$ value calculated based on Fisher's exact test. A) Canonical pathways associated with modified proteins acting in energy metabolism. B) Canonical pathways associated with modified proteins acting in quality control and stress response

Figure 4: Glycolysis pathway related metabolites alterations in senescent fibroblasts

Glycolysis pathway related metabolites profiling in young (FY) and senescent WI-38 fibroblasts (FS). For the box plots, the top and bottom of the boxes represent the 75th and 25th percentile, respectively. The solid bar across the box represents the median value, while the + is the mean. The $\mathrm{Y}$ axis is the median scaled value (relative level). The fold change and the corresponding $\mathrm{p}$ value in senescent cells relative to their young counterpart is indicated in each plot.

Figure 5: Tricarboxylic acid (TCA) cycle related metabolites alterations in senescent fibroblasts Tricarboxylic acid (TCA) cycle related metabolites profiling in young (FY) and senescent WI-38 fibroblasts (FS). For the box plots, the top and bottom of the boxes represent the $75^{\text {th }}$ and $25^{\text {th }}$ percentile, respectively. The solid bar across the box represents the median value, while the + is the mean. Statistical outlier is represented by a circle. The $\mathrm{Y}$ axis is the median scaled value (relative level). The fold change and the corresponding $\mathrm{p}$ value in senescent cells relative to their young counterpart is indicated in each plot. 


\section{Figure 1}

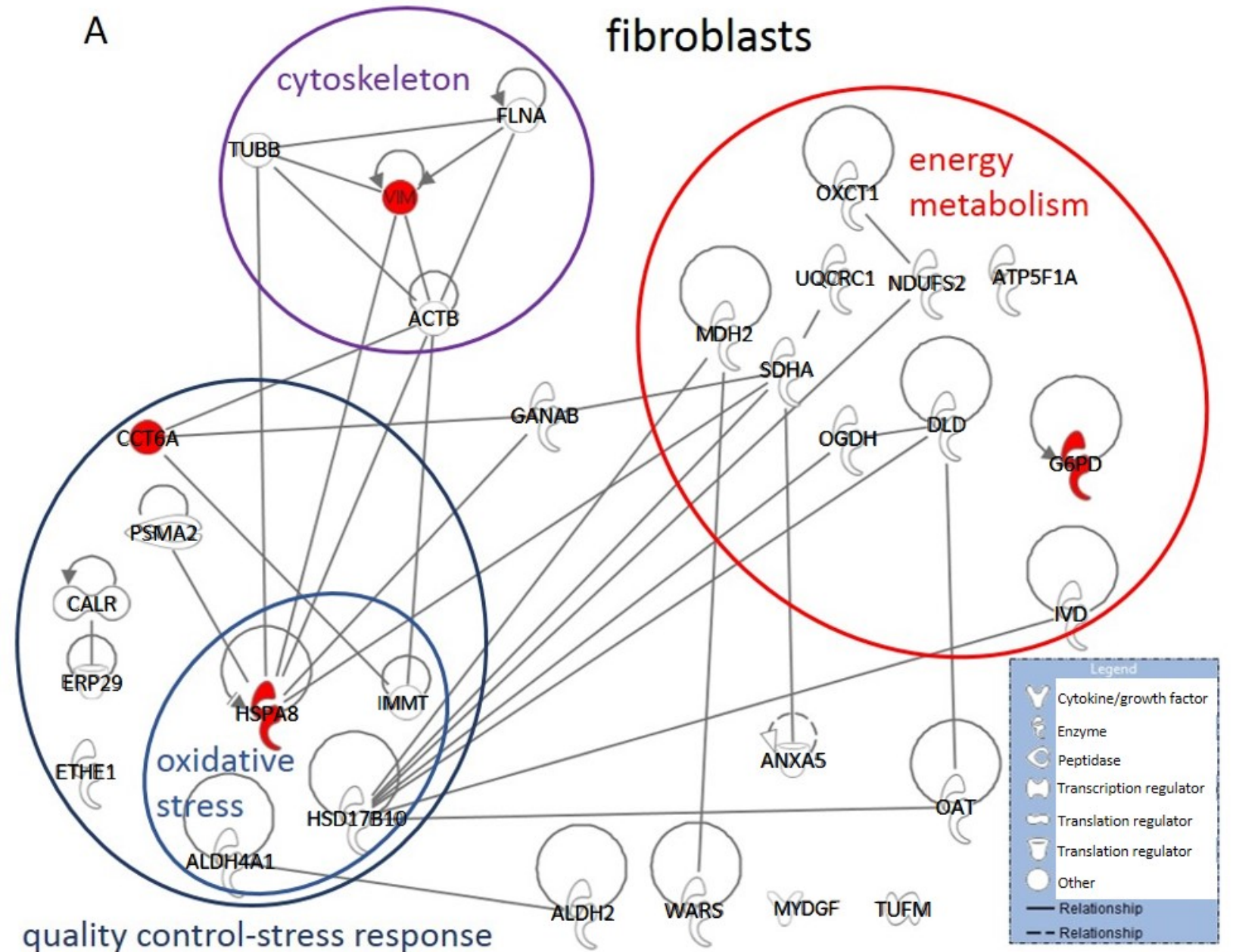

B

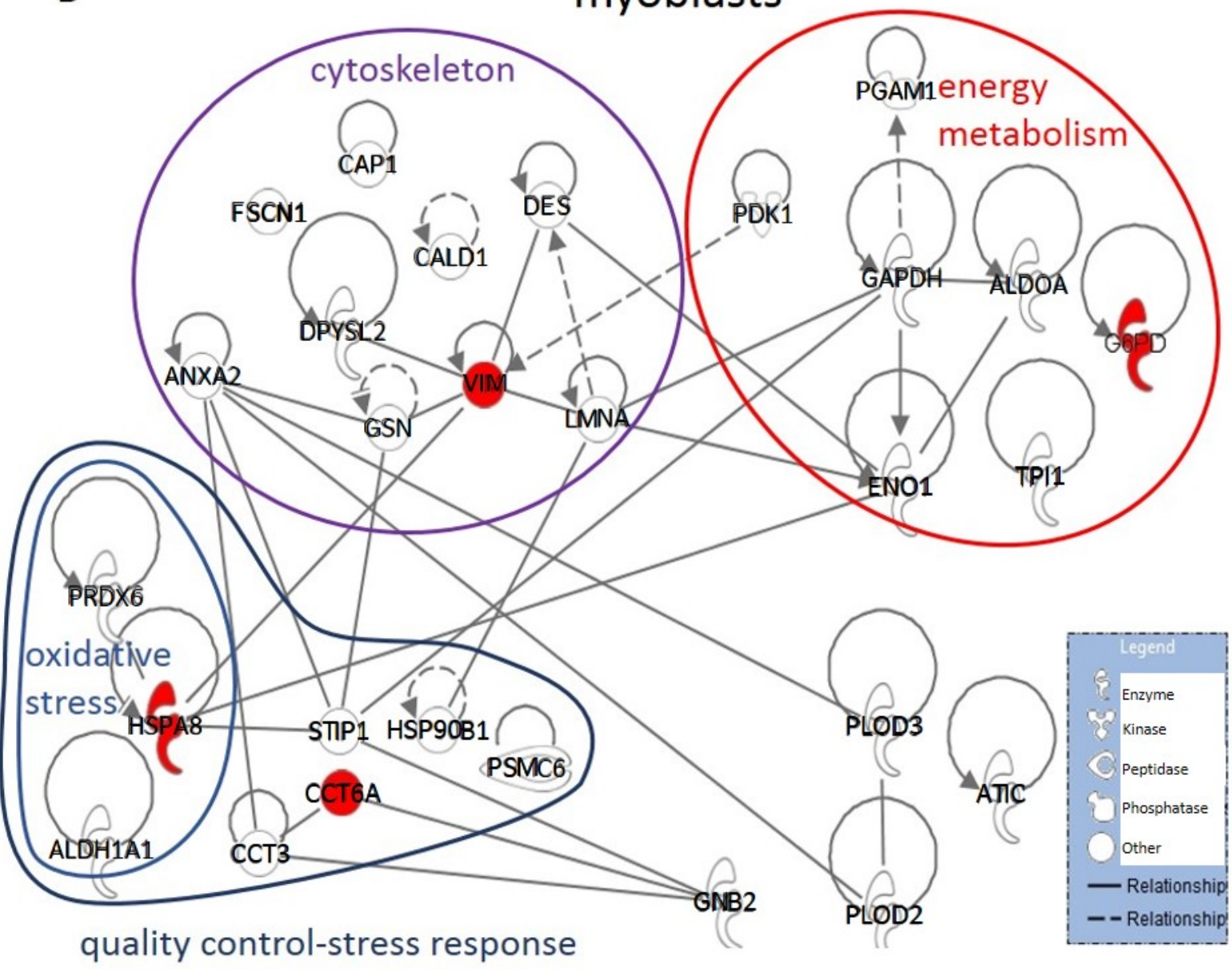




\section{Figure 3}
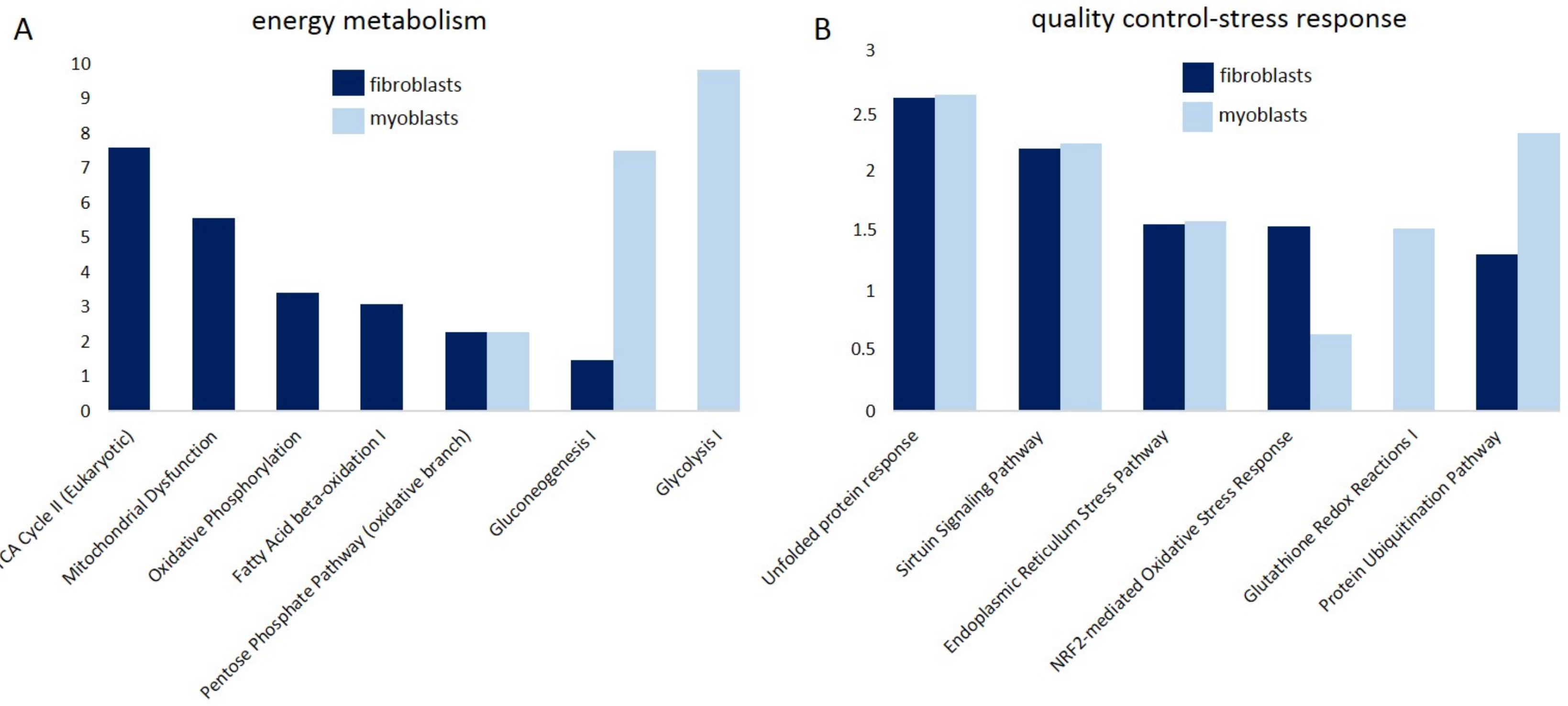


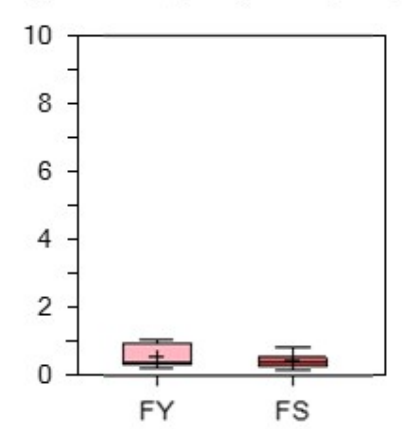

6-phosphogluconate

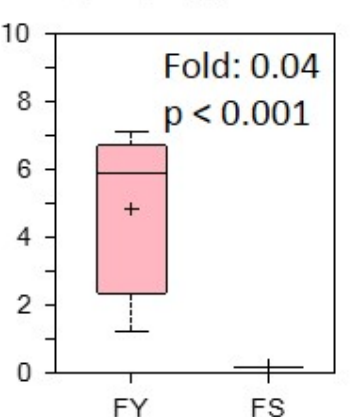

6-phosphogluconate
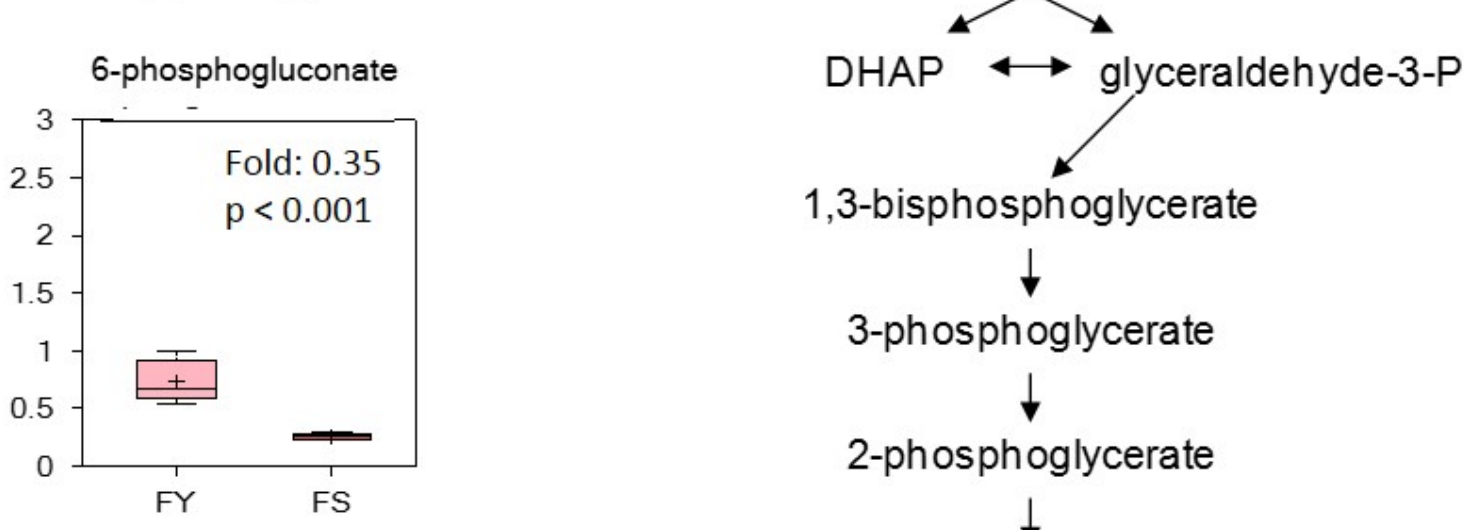

phosphoenolpyruvate (PEP)

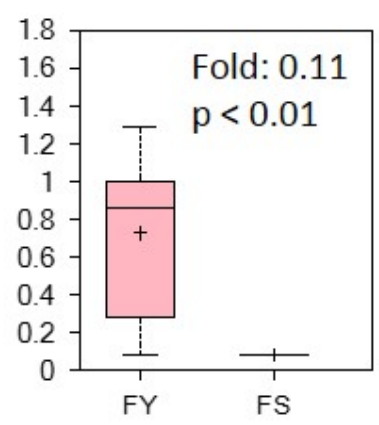

2-phosphoglycerate

$$
\downarrow
$$

phosphoenolpyruvate
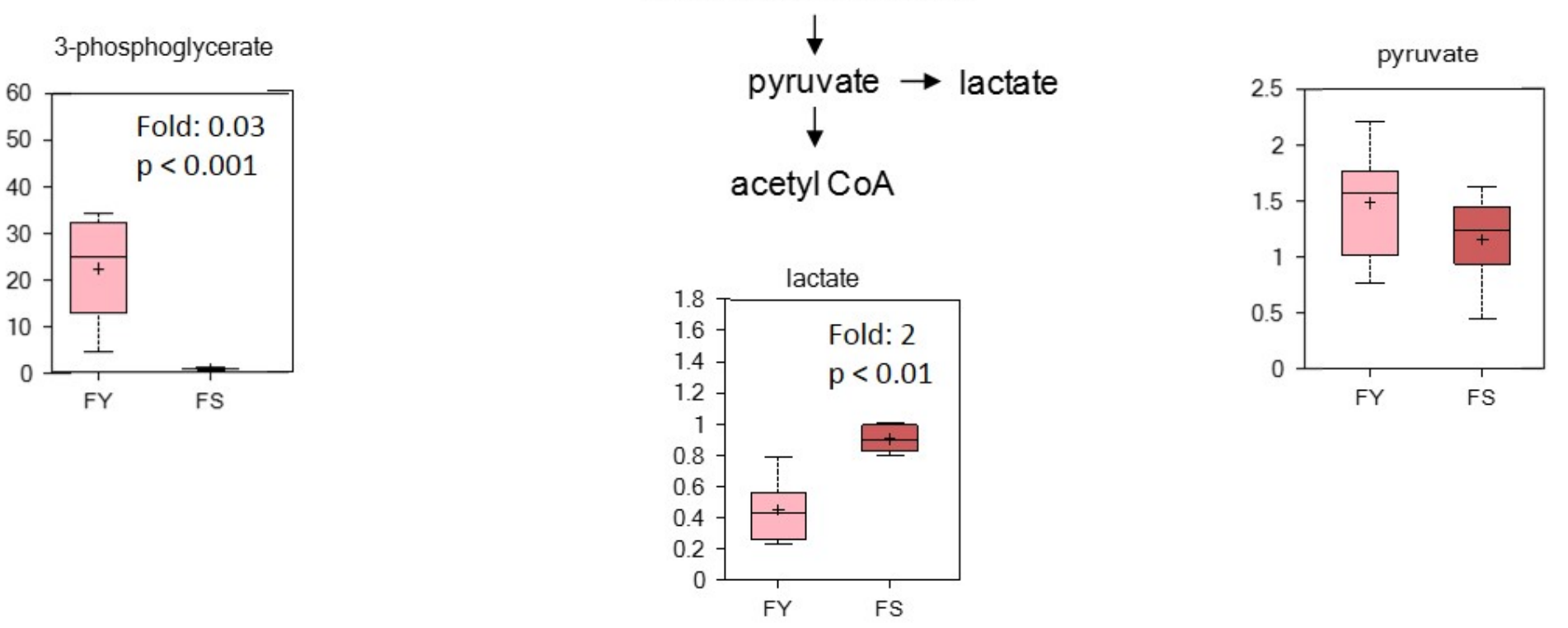

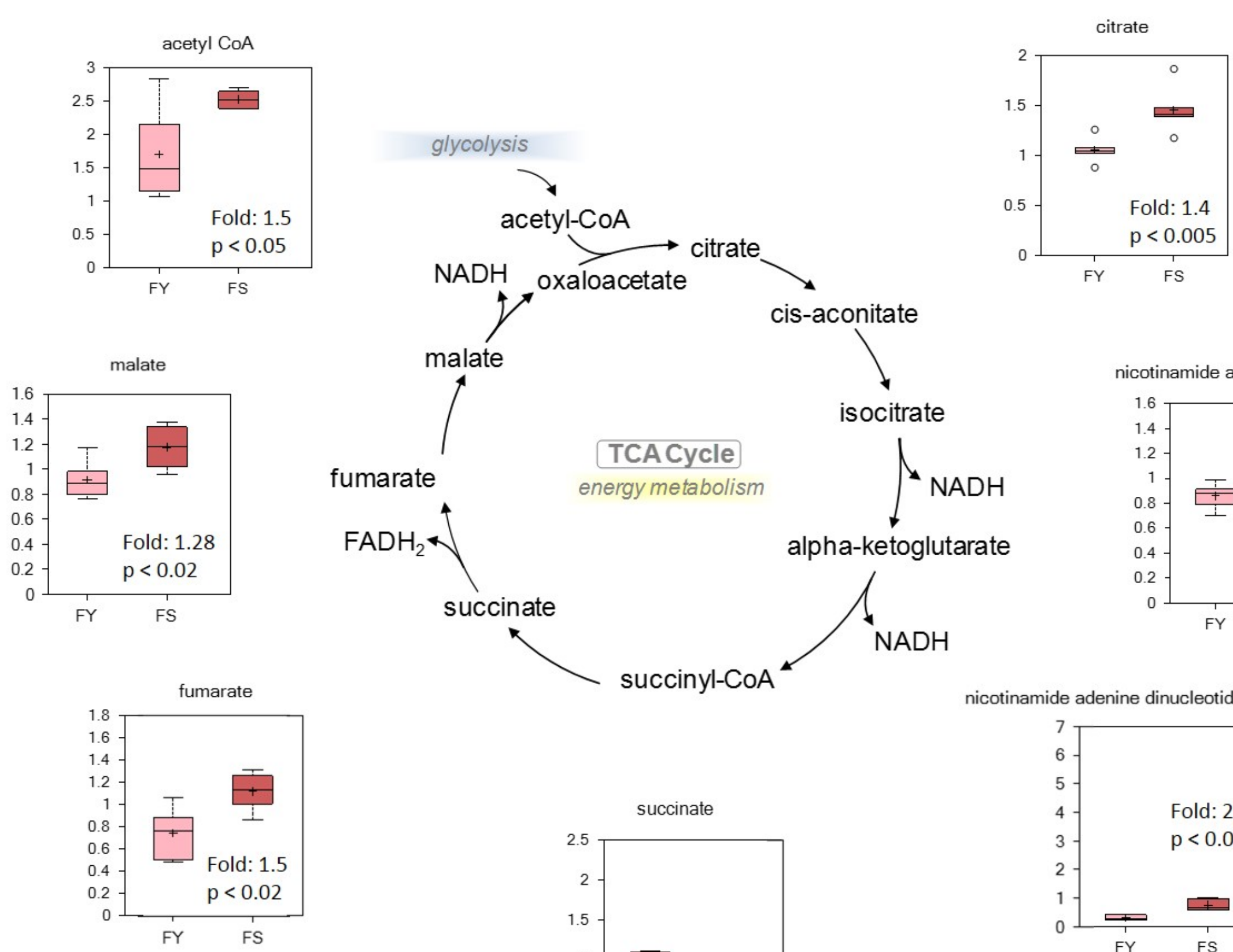

cis-aconitate
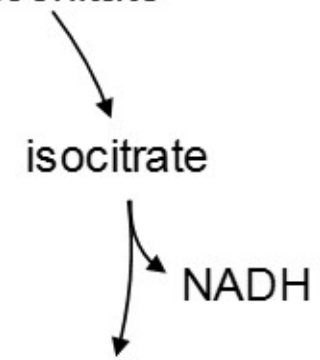

alpha-ketoglutarate

$\mathrm{NADH}$ nicotinamide adenine dinucleotide $(\mathrm{NAD}+)$

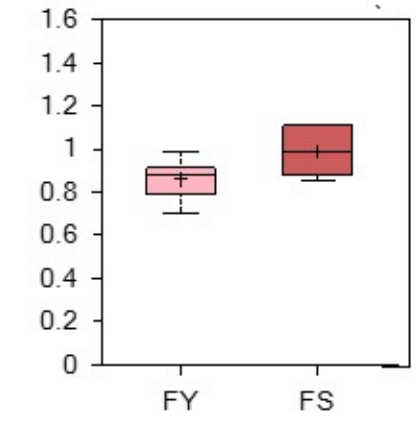

nicotinamide adenine dinucleotide reduced (NADH)

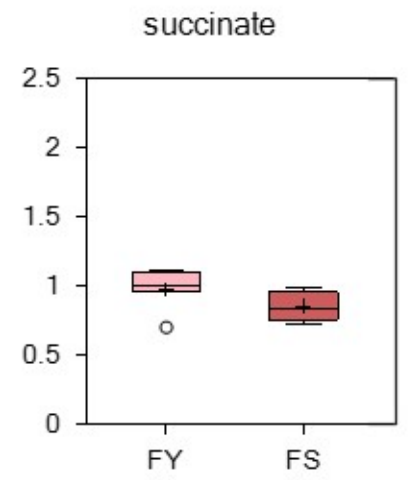

